
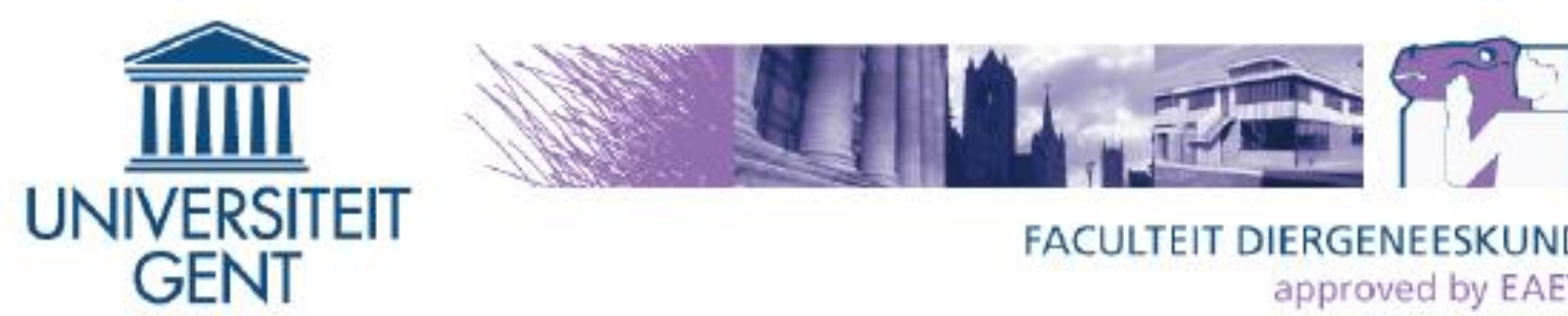

\title{
Influence of mycotoxin binders on the oral bioavailability of doxycycline in pigs
}

Thomas De Mil${ }^{1}, \underline{\text { Mathias Devreese }}{ }^{1}$, Sarah De Saeger ${ }^{2}$, Mia Eeckhout ${ }^{3}$ and Siska Croubels ${ }^{1}$

${ }^{1}$ Department of Pharmacology, Toxicology and Biochemistry, Faculty of Veterinary Medicine

2 Department of Bioanalysis, Faculty of Pharmaceutical Sciences

${ }^{3}$ Department of Applied Biosciences, Faculty of Bioscience Engineering

\section{MYTOX}

$\underline{\text { www.mytox.be }}$ 
Introduction: Mycotoxin binders

\section{Mycotoxin detoxifiers}

Mycotoxin binders
Mycotoxin modifiers

Clay based

Yeast based 


\section{Introduction: Mycotoxin binders}

- Feed additive: as mycotoxin binder

Recommended level: $1-2 \mathrm{~g} / \mathrm{kg}(0.1 \%-0.2 \%)$

COMMISSION REGULATION (EC) No 386/2009

of 12 May 2009

amending Regulation (EC) No 1831/2003 of the European Parliament and of the Council as regards the establishment of a new functional group of feed additives

(Text with EEA relevance)

- Feed additive: for technological purposes

Maximally $20 \mathrm{~g}$ bentonite/kg feed (2\%)

REGULATION (EC) No 1831/2003 OF THE EUROPEAN PARLIAMENT AND OF THE COUNCIL of 22 September 2003

on additives for use in animal nutrition

(Text with EEA relevance) 


\section{Introduction: Doxycycline (DOX)}

- Tetracycline antibiotic

- Mycoplasma spp., Actinobacillus pleuropneumoniae, Bordetella bronchiseptica, etc.

- Oral bioavailability is lower in fed animals
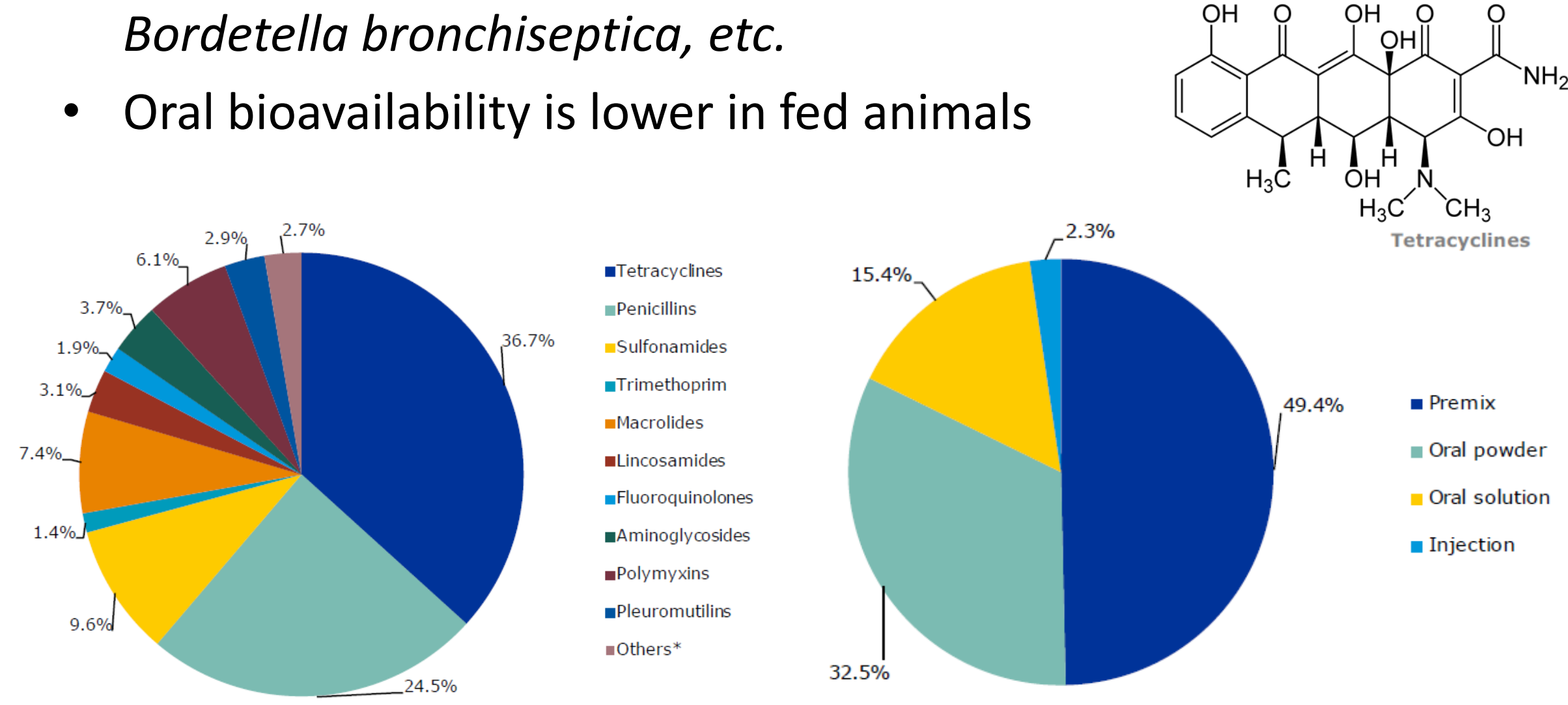

-Tetracyclines - Penicillins

-Sulfonamides

-Trimethoprim

-Macrolides

nLincosamides

-Fluoroquinolones

-Aminoglycosides

aPolymyxins

aPleuromutilins

mothers* 


\section{Aims}

Mycotoxin binders: feed additives

Antimicrobials (doxycycline): Oral administration via feed/drinking water
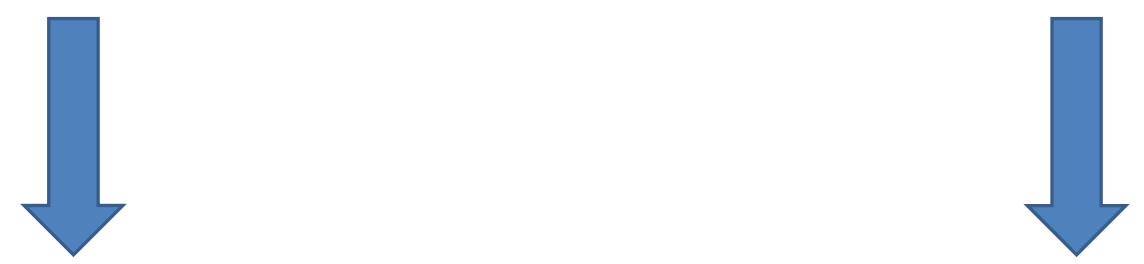

Non-specific interaction in the GIT?

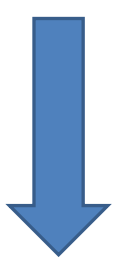

Interaction depends on

- inclusion rate?

- prandial status? 


\section{Experiment 1: Study design}

Fasted animals, single oral bolus:

- Daily dosis of mycotoxin binder ( $0.2 \%$ inclusion rate)

- DOX (10 mg/kg BW)

Control

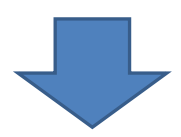

\section{resis}

\section{Clay 1}

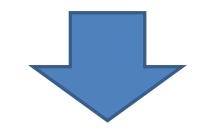

4

Clay 2

min

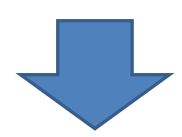

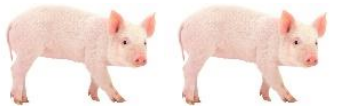

\section{rats}
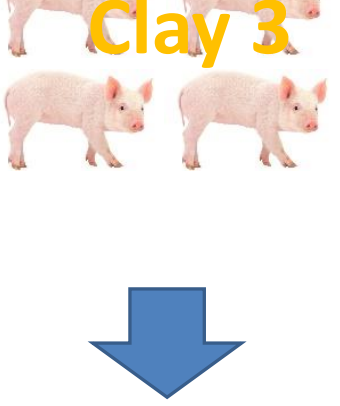

Yeast 1
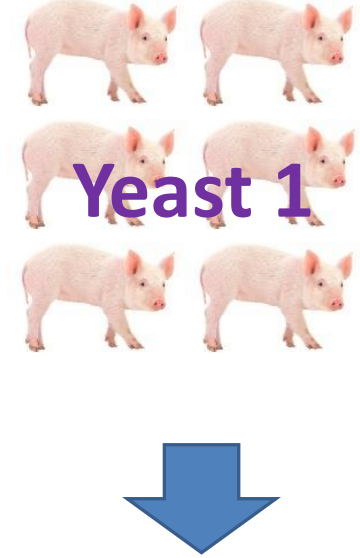

Blood sampling at different time points after DOX administration

LC-MS/MS analysis of DOX in plasma

Comparing PK 


\section{Experiment 1: Results}

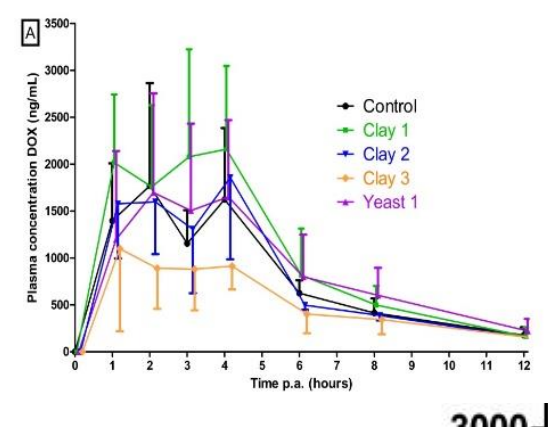

$$
\text { Relative } F(\%)=\frac{A U C \text { test group }}{A U C \text { control }} \times 100
$$

\begin{tabular}{|c|c|c|c|c|c|}
\hline Treatment group & Control & Clav 1 & Clav 2 & Clav 3 & Yeast 1 \\
\hline $\mathrm{AUC}_{0-12 \mathrm{~h}}(\mathrm{~h} \cdot \mu \mathrm{g} / \mathrm{mL})$ & $9.60 \pm 3.49$ & $12.29 \pm 4.57$ & $9.73 \pm 2.35$ & $6.37 \pm 2.63$ & $10.72 \pm 5.59$ \\
\hline $\mathrm{AUC}_{0 \text {-inf }}(\mathrm{h} \cdot \mu \mathrm{g} / \mathrm{mL})$ & $10.56 \pm 3.97$ & $12.90 \pm 4.84$ & $10.43 \pm 2.42$ & $7.54 \pm 2.67$ & $11.78 \pm 6.04$ \\
\hline Relative F (\%) & $100.00 \pm 37.61$ & $122.31 \pm 45.87$ & $98.91 \pm 22.95$ & $71.50 \pm 25.34$ & $111.69 \pm 57.28$ \\
\hline$T_{\max }(h)$ & $2.00 \pm 1.10$ & $2.83 \pm 1.47$ & $2.50 \pm 1.64$ & $2.17 \pm 1.47$ & $2.50 \pm 1.22$ \\
\hline$C_{\max }(\mu \mathrm{g} / \mathrm{mL})$ & $2.01 \pm 0.91$ & $2.46 \pm 0.90$ & $2.12 \pm 0.77$ & $1.31 \pm 0.75$ & $1.93 \pm 1.06$ \\
\hline$k_{\mathrm{el}}(1 / \mathrm{h})$ & $0.23 \pm 0.08$ & $0.29 \pm 0.05$ & $0.22 \pm 0.04$ & $0.21 \pm 0.13$ & $0.23 \pm 0.06$ \\
\hline$T_{1 / 2 \mathrm{el}}(\mathrm{h})$ & $4.83 \pm 1.74$ & $3.57 \pm 0.52$ & $4.69 \pm 0.87$ & $6.59 \pm 3.95$ & $4.60 \pm 1.11$ \\
\hline $\mathrm{Vd} / \mathrm{F}(\mathrm{L} / \mathrm{kg})$ & $4.77 \pm 1.35$ & $3.07 \pm 1.12$ & $4.66 \pm 1.14$ & $9.26 \pm 5.81$ & $5.36 \pm 3.92$ \\
\hline $\mathrm{Cl} / \mathrm{F}(\mathrm{L} / \mathrm{h} / \mathbf{k g})$ & $1.09 \pm 0.46$ & $0.88 \pm 0.33$ & $1.00 \pm 0.23$ & $1.45 \pm 0.43$ & $1.15 \pm 0.76$ \\
\hline
\end{tabular}

\section{Time p.a. (hours)}




\section{Experiment 2: Study design}

Fasted animals, single oral bolus:

- Daily dosis of mycotoxin binder ( $\mathbf{1 \%}$ inclusion rate)

- DOX (10 mg/kg BW)

\section{Control}

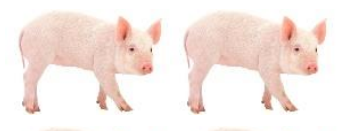

Clay 1
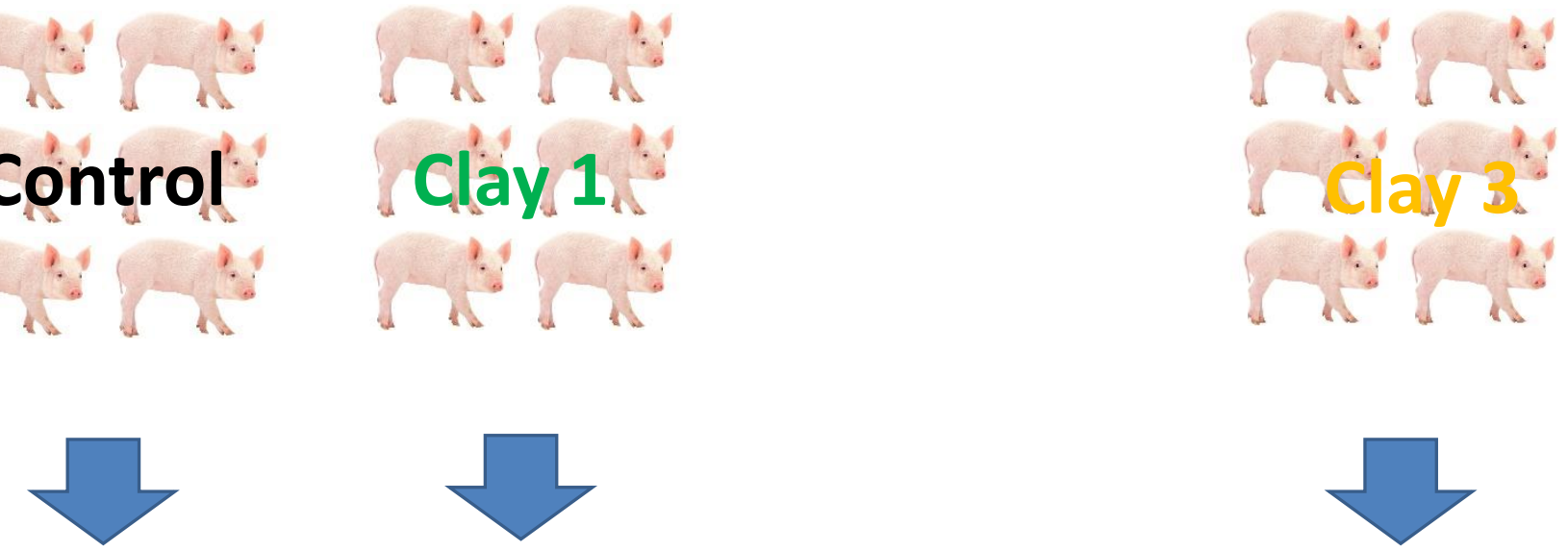

Blood sampling at different time points after DOX administration

LC-MS/MS analysis of DOX in plasma

Comparing $\mathrm{PK}$ 


\section{Experiment 3: Study design}

\section{Continuous administration in the feed:}

- Mycotoxin binder (0.2\% inclusion rate)

- DOX: $270 \mathrm{mg} / \mathrm{kg}$ feed ( $10 \mathrm{mg} / \mathrm{kg} \mathrm{BW}$ )

Control

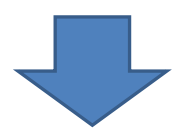

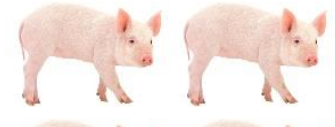

Clay 1

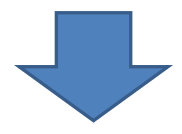

14

Clay 2

min

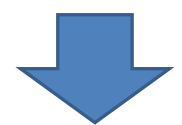

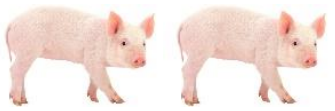

ndiay 3
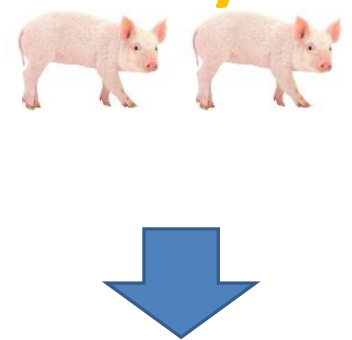

Yeast 1

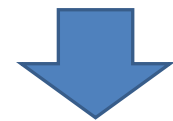

Blood sampling at different time points after DOX administration

LC-MS/MS analysis of DOX in plasma

Comparing $\mathrm{PK}$ 


\section{Experiment 3: Results}

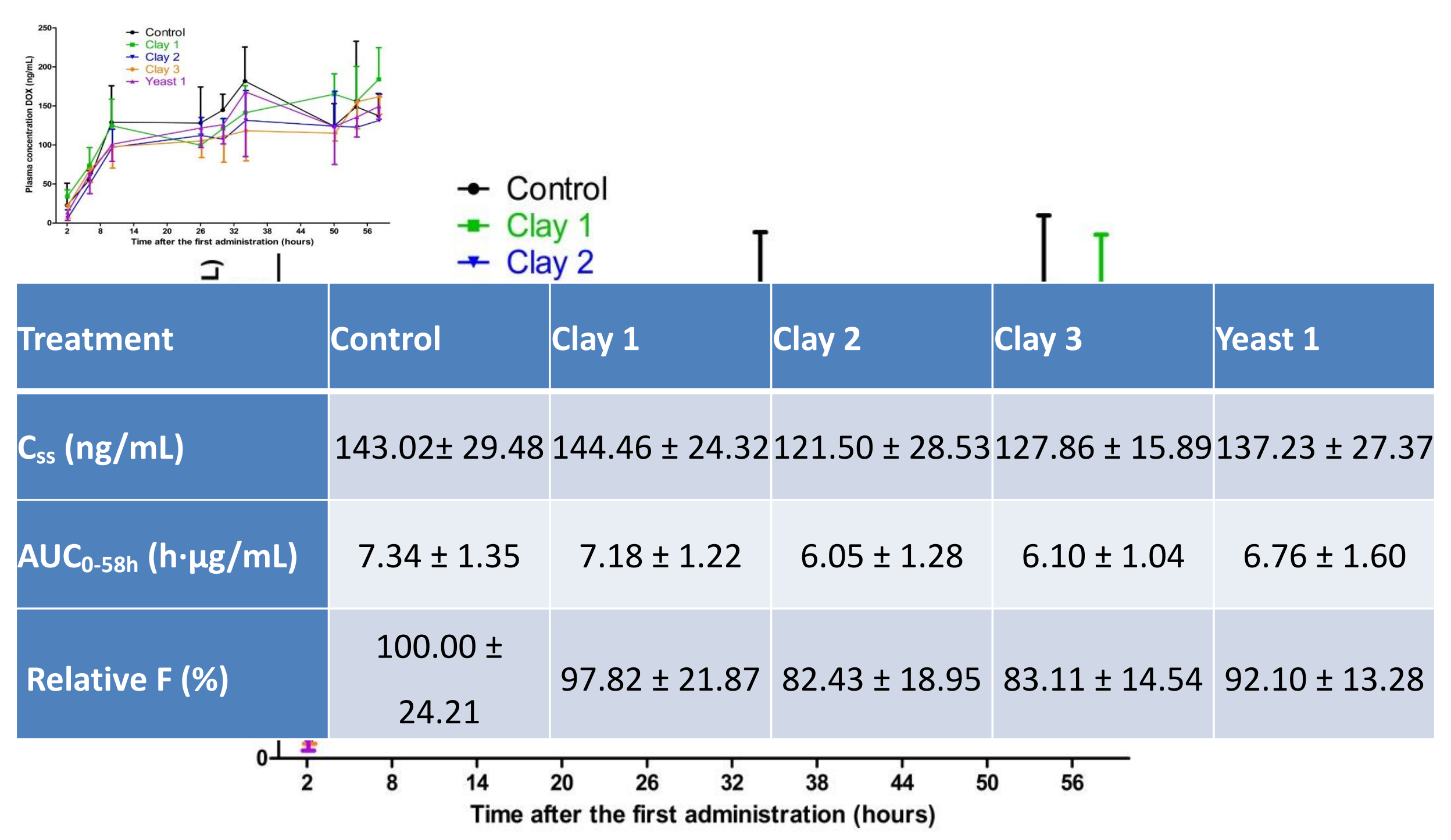




\section{Discussion \& Conclusions}

- Interaction possible at high dose, in fasted animals

- No interactions observed at low dose

- Confirms in vitro results using feed containing buffered matrix (Poster 17)

- Bolus mo

- EFSA (201

- Highe:

- Steadi days $\mathrm{s}$

- Further ir

De Mil et al

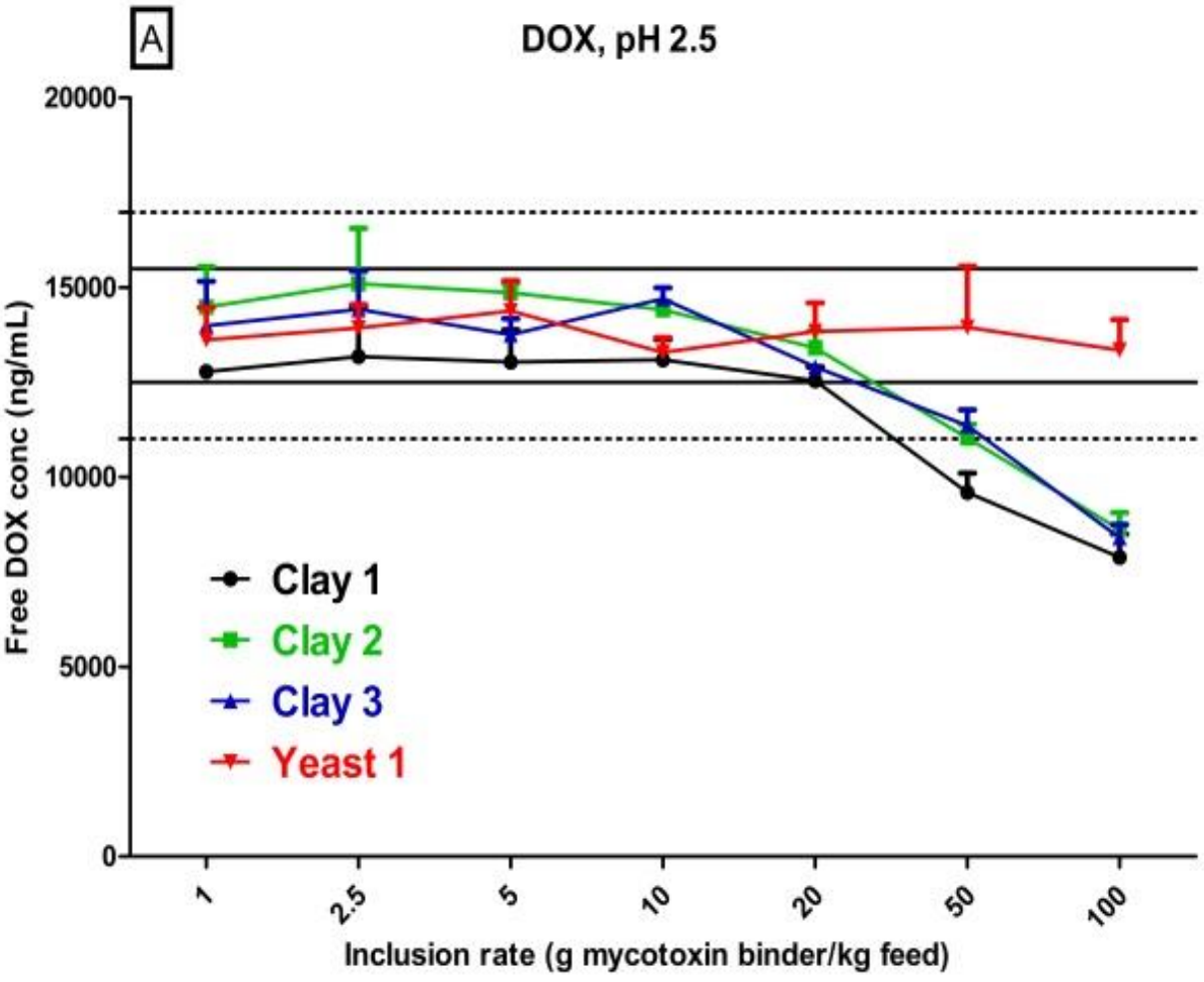

roduct in feed, 5

EFSA Journal 2010; 8(7):1693

NTIFIC OPINION

of guidelines for the assessment of additives ances for reduction of the contamination of by mycotoxins, ${ }^{1}$

ts or Substances used in Animal Feed (FEEDAP) ${ }^{2,3}$ 


\section{Acknowledgements}
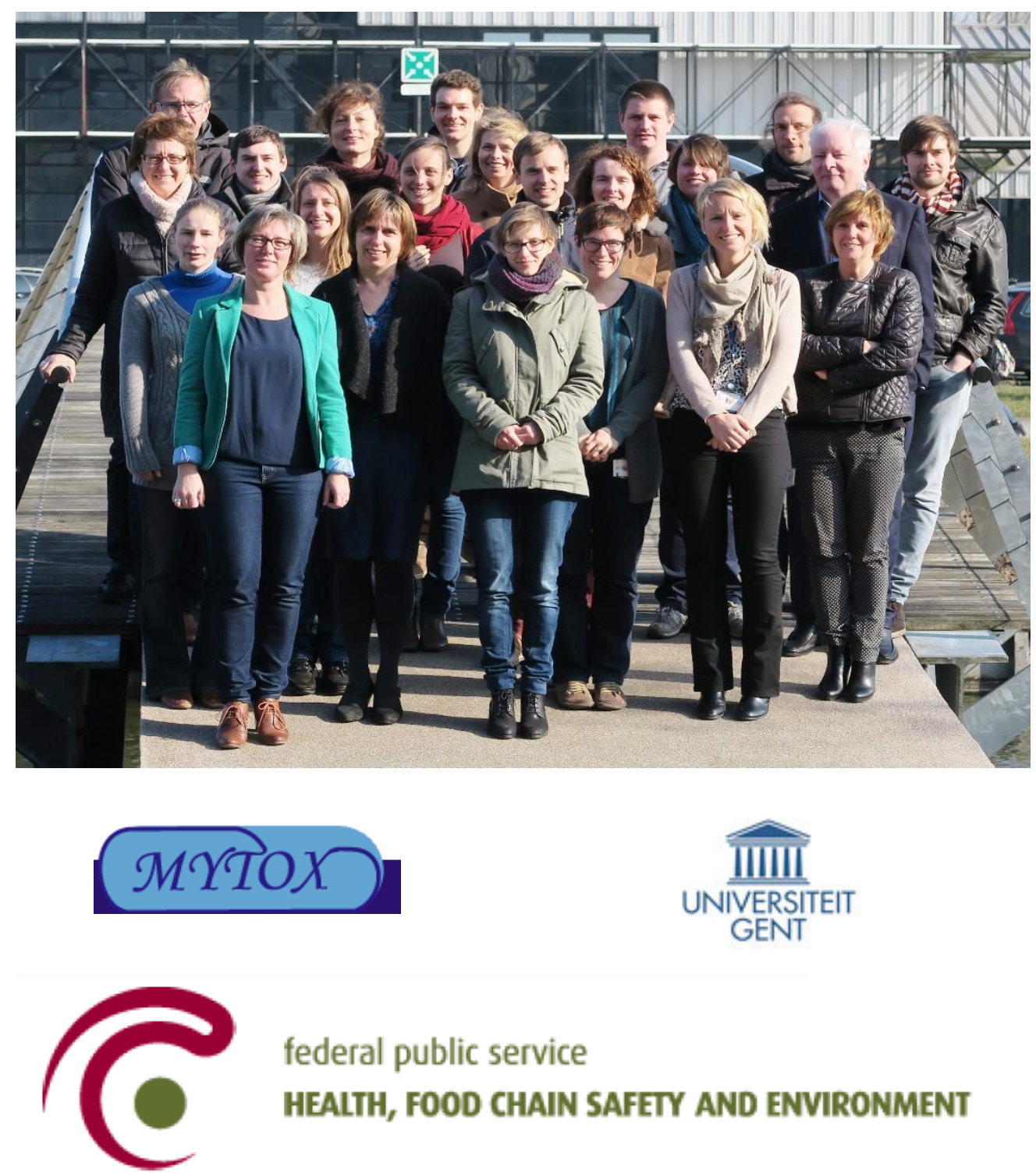

$$
\underset{\substack{\text { UNIVERSITEIT } \\ \text { GENT }}}{\widehat{\text { IIIII }}}
$$

federal public service

HEALTH, FOOD CHAIN SAFETY AND ENVIRONMENT 